\title{
Tuberculose e TBMR: mecanismos imunológicos e novas ferramentas de controle da doença
}

DOI: 10.3395/reciis.v2i1.132pt

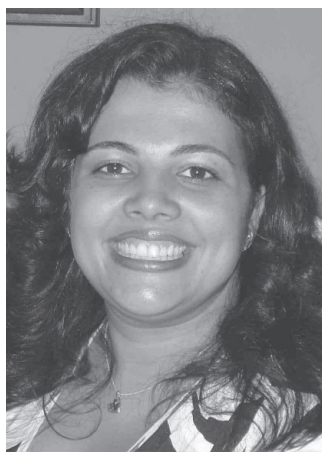

Roberta Olmo

\section{Pinheiro}

Laboratório de Hanseníase, Instituto Oswaldo Cruz, Fundação Oswaldo Cruz, Rio de Janeiro, Brasil rolmo@ioc.fiocruz.br

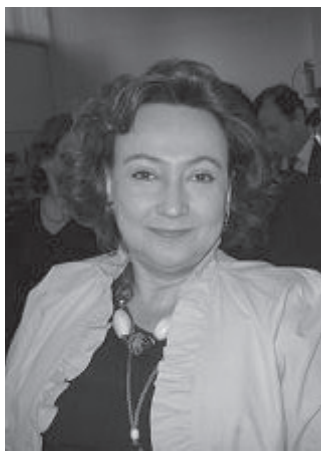

Margareth Pretti Dalcolmo Centro de Referência Professor Hélio Fraga, UFRJ, Rio de Janeiro, Brasil margareth.dalcolmo@ saude.gov.br

\section{Elizabeth Pereira Sampaio}

Instituto Oswaldo Cruz, Fundação Oswaldo Cruz, Rio de Janeiro, Brasil esampaio@ioc.fiocruz.br

\section{Resumo}

Estima-se que um terço da população mundial esteja infectada com o Mycobacterium tuberculosis. Em 2005, a Organização Mundial da Saúde estimou em oito milhões o número de mortes causadas por tuberculose em todo o mundo. Os indicadores oficiais apontam declínio da doença no último século; entretanto, desde 1990 este declínio vem apresentando sinais de reversão. Apesar da existência de medicamentos efetivos no controle da tuberculose, os casos de resistência a múltiplas drogas têm aumentado em todo mundo. Recentemente, esse problema se agravou com a emergência de cepas extremamente resistentes a drogas, denominadas pela OMS como XDRTB (extensively drug resistant tuberculosis). A vacina existente contra a doença, o BCG, é capaz de prevenir as formas graves de tuberculose em crianças; no entanto, sua eficácia em adultos é altamente variável e foi demonstrado que a revacinação em jovens e adultos não aumenta o nível de proteção. Diversos estudos demonstraram que as células hospedeiras desenvolveram mecanismos imunorregulatórios e micobactericidas na tentativa de conter a infecção e que falhas nesses mecanismos seriam responsáveis pelo avanço da doença. No presente artigo procura-se revisar os dados relacionados à resposta imune em pacientes com tuberculose e tuberculose multirresistente (TBMR) e como esses achados podem contribuir para o desenvolvimento de novas estratégias de diagnóstico e/ou vacinas para o controle da doença.

\section{Palavras-chave}

Tuberculose, multirresistente, diagnóstico, IFN- $\gamma$, mecanismos imunológicos 


\section{Introdução - controle da tuberculose}

O Mycobacterium tuberculosis, em conjunto com o HIV são os dois principais agentes responsáveis pelas mortes causadas por doenças infecciosas no mundo (WHO, 1996). Em 2005, a Organização Mundial da Saúde estimou oito milhões de novos casos e 1,6 milhões de mortes causadas por tuberculose em todo o mundo (WHO, 2007). Apesar dos indicadores oficiais terem relatado o declínio da doença no último século, desde 1990 este declínio vem apresentando sinais de reversão (LOCHT et al., 2007).

As Américas respondem por 4\% das notificações de tuberculose em nível global, notificando em 2003, 227.551 casos de tuberculose. No Brasil, em 2003, foram notificados 83.575 casos novos, correspondendo ao coeficiente de incidência de 47,3/100.000 habitantes, variando de 18,7/100.000 no Tocantins a 79,6/100.000 no Rio de Janeiro (WHO, 2005). O Brasil encontra-se incluído entre os 22 países que concentram $80 \%$ dos casos, em números absolutos, ocupando o $15^{\circ}$ lugar neste ranking (WHO, 2005).

Estima-se que 1/3 da população mundial esteja infectada com o $M$. tuberculosis. No entanto, a maior parte dos indivíduos infectados não desenvolve qualquer sinal de doença em toda sua vida, mantendo uma infecção latente. No entanto, o diagnóstico dos indivíduos com infecção latente é importante, uma vez que estes apresentam risco de desenvolver a doença, especialmente se expostos a condições que favoreçam o desenvolvimento da micobactéria, como a co-infecção com HIV ou ainda quando em utilização de imunossupressores (LOTCH et al., 2007).

O controle eficiente da tuberculose está associado ao diagnóstico tanto da doença quanto da infecção latente, como no tratamento efetivo e/ou vacinas para prevenir a progressão dos indivíduos com a infecção latente para a doença ativa.

Para a melhoria das condições relacionadas ao controle da tuberculose, foi introduzida a estratégia DOTS (Directly Observed Treatment Short Course), que é pautada em cinco pilares considerados essenciais para o controle da doença: compromisso político, detecção de casos por baciloscopia, esquemas de tratamento padronizados e tratamento diretamente supervisionado, suprimento regular e ininterrupto dos medicamentos padronizados e sistema de registro e notificação de casos (WHO, 2006). A estratégia DOTS tem sido adotada por mais de 150 países (RAVIGLIONE et al., 2002), apesar de um quarto da população mundial não ter ainda acesso a esse serviço.

Na maioria dos países com elevada prevalência, o diagnóstico da tuberculose ativa é baseado na anamnese clínica seguido pela identificação do bacilo em esfregaços utilizando microscopia ótica. Na tuberculose pulmonar, a tosse não produtiva é o sintoma mais comum no início da doença. Com o desenvolvimento da infecção, o escarro começa a ser produzido quando aumenta a inflamação e a necrose no tecido pulmonar. Devido a isso, a baciloscopia é o método prioritário de diagnóstico e controle durante o tratamento da tuberculose (TEIXEIRA et al., 2007). No entanto, apesar do baixo custo, a sensibilidade deste método é altamente variável, chegando a índices menores que 20\% (STEINGART et al., 2006; URBANZIK, 1985).

O teste intradérmico utilizando o derivado protéico purificado (PPD) é pouco específico, uma vez que o PPD contém diversos antígenos micobacterianos que são compartilhados com outros microrganismos, incluindo o BCG.

Desde 1982, as drogas de primeira escolha no tratamento da tuberculose no Brasil são a isoniazida e a rifampicina por seis meses em associação com a pirazinamida nos dois primeiros meses. Cabe ressaltar que os medicamentos são fornecidos pelo governo, sem ônus ao paciente; entretanto, o tratamento é baseado em auto-administração, o que acaba favorecendo os casos de abandono do tratamento, quer em decorrência do aparecimento de reações adversas ou simplesmente devido ao fato do paciente sentir-se melhor e acreditar não precisar mais do tratamento.

A tuberculose multirresistente (TBMR) é descrita como sendo aquela resistente a isoniazida e rifampicina in vitro (YEW et al., 1995). No Brasil, considera-se TBMR a situação na qual o paciente é resistente à rifampicina e à isoniazida e a um terceiro fármaco dos esquemas padronizados (DALCOLMO et al., 2007).

Em um estudo multicêntrico visando avaliar a eficácia dos regimes terapêuticos para TBMR foi observado que o tratamento irregular ou incompleto foi o indicador mais importante da multirresistência (DALCOLMO et al., 1999). Outros fatores como a presença de cavitações na radiologia, e a co-infecção com HIV também parecem ser importantes para o desenvolvimento das formas resistentes (GRANICH et al., 2005; VANACORE et al., 2004). Entretanto, no Brasil, a taxa de co-infecção em pacientes com HIV/aids tem sido estimada em 3-4\% dos casos. Entre os pacientes com aids, no momento do diagnóstico, o percentual de co-infecção com tuberculose, que foi de 30\% nos anos 1990, apresenta uma tendência de queda, fato que alguns autores associam com a introdução da quimioterapia antiviral (HIJJAR et al., 2005).

Uma vez que os índices de abandono do tratamento estão associados à ocorrência de TBMR, diversos estudos têm procurado avaliar o nível de adesão à terapia em pacientes com tuberculose. No Rio de Janeiro dois estudos encontraram taxas de falta de adesão ao tratamento de 30,5 e 28,9\%, respectivamente (DINIZ et al., 1995; KRITSKI et al., 2002). No estado de São Paulo, onde há elevados índices de TBMR, DEHEINZELIN et al. (1996) demonstraram uma taxa de abandono do tratamento de $33 \%$.

Dados da Organização Mundial da Saúde apontam que no Brasil, a prevalência nacional de resistência adquirida a qualquer droga é menor do que $20 \%$ e a resistência a múltiplas drogas é menor do que 10\% (WHO, 2004). No entanto, variações regionais devem ser levadas em consideração. Recentemente, BALIZA et al. (2008) des- 
creveram que na cidade de Cabo de Santo Agostinho, em Pernambuco, do total de casos de TB, 14\% eram multirresistentes. Já outro estudo realizado na cidade de São Paulo demonstrou taxa de resistência de 15,5\%. Neste estudo, entre os casos previamente tratados, a prevalência de resistência foi de $27 \%$ e a resistência a múltiplas drogas foi de 16,7\% (TELLES et al., 2005).

Os tipos de resistência do $M$. tuberculosis podem ser classificados em: natural (decorrente de mutação espontânea, independente da exposição prévia a fármacos e proporcional ao número de bacilos); inicial (observada no momento em que o paciente se apresenta para o tratamento. Inclui pacientes com resistência primária ou adquirida, sobre os quais não se conhece informações de tratamentos anteriores); primária (observada em pacientes não tratados anteriormente, infectados por uma fonte com forma resistente) e adquirida ou secundária (resultante de uso prévio de medicação de forma inadequada) (MITCHISON, 2005). Estudos anteriores demonstraram que pacientes com TBMR transmitem e causam a doença em pessoas susceptíveis, da mesma forma que o fazem os doentes sensíveis às drogas (SNIDER et al., 1985; VALWAY et al., 1994). Por ser facilmente contagiosa, com a conseqüente disseminação de bacilos resistentes na população, o controle da doença torna-se deficiente.

Encontra-se em desenvolvimento no Brasil o II Inquérito nacional de resistência aos fármacos anti-TB que vai atualizar as taxas de multirresistência no país. Espera-se que, apesar da grande variação entre algumas regiões, se encontre taxas de resistência associadas à Rifampicina + Isoniazida ainda em níveis muito baixos. Em revisão recente, ZAGER et al. (2008) descreveram que a prática de expressar a prevalência de TBMR como a proporção dos casos encontrados resistentes não reflete adequadamente a ocorrência de MR na comunidade. Para obter um painel adequado da incidência ou prevalência da doença em uma determinada população, os autores sugerem que sejam incluídos os casos de multirresistência que apareceram em casos previamente tratados.

O tratamento recomendado para TBMR é menos efetivo, menos eficaz, mais longo, mais tóxico e mais caro do que o tratamento da tuberculose sensível. Requer o uso de medicamentos de segunda e de terceira linhas, por período entre 18 a 24 meses (ISEMAN, 1993; ZIGNOL et al., 2006, DALCOLMO et al., 2007).

Uma nova ameaça para o controle da tuberculose é o aparecimento de cepas extremamente resistentes (XDRTB). A XDR-TB é definida como sendo a resistência à rifampicina, isoniazida, um medicamento injetável de segunda linha (amicacina, kanamicina, capreomicina) e uma fluoroquinolona (ESPINAL et al., 2001; CDC, 2007).

Apesar do conhecimento acumulado sobre os mecanismos imunológicos associados à patogênese da doença, ainda não há uma completa compreensão sobre os mecanismos de sinalização, as respostas transcripcionais do hospedeiro, a adaptação da bactéria ao hospedeiro ou interações célula-célula que se seguem à infecção com $M$. tuberculosis. Assim, alguns estudos têm procurado avaliar os mecanismos imunológicos associados à infecção e ao desenvolvimento de resistência em alguns indivíduos.

\section{Mecanismos imunológicos associados ao controle da tuberculose e da TBMR}

A tuberculose é predominantemente uma doença pulmonar, sendo esta responsável por $80 \%$ dos casos notificados no Brasil; entretanto, o bacilo pode invadir as correntes sanguínea e linfática e disseminar-se pelo organismo, causando doença virtualmente em qualquer órgão do corpo humano. Logo após a infecção primária por partículas aéreas, os macrófagos alveolares e as células dendríticas que fagocitaram o $M$. tuberculosis migram através do sistema linfático em direção ao linfonodo regional e formam o complexo de Ghon. Ao mesmo tempo as células fagocíticas podem penetrar no parênquima pulmonar, iniciando um foco inflamatório ao redor do microorganismo, levando à formação do granuloma num processo coordenado por linfócitos $\mathrm{T}$ (TEIXEIRA et al., 2007). Estudos envolvendo a análise da interação entre $M$. tuberculosis e células apresentadoras de antígeno demonstrou que as células dendríticas não permitem o crescimento intracelular de $M$. tuberculosis, ao contrário do que é observado em macrófagos (HERMANN et al., 2006). Nos macrófagos, a micobactéria reside nos fagossomas iniciais e escapa do sistema imune por inibir a maturação dos fagossomas e a fusão com os lisossomas (RUSSELL, 2003; 2007). O M. tuberculosis produz lipídios que mimetizam os fosfatidilinositóis de mamíferos e com isso inibe as vias dependentes de fosfatidilinositol 3-fosfato em macrófagos infectados (VERGNE et al., 2004).

A imunidade adaptativa contra o $M$. tuberculosis envolve principalmente as células $\mathrm{T} \mathrm{CD}^{+}$. A importância das células $\mathrm{T} \mathrm{CD}^{+}$produtoras da citocina IFN- $\gamma$ na resistência primária contra o $M$. tuberculosis já foi descrita (COOPER et al., 1997). No entanto, as células $\mathrm{T} \mathrm{CD}^{+}$parecem ter um importante papel no controle da doença, uma vez que a perda das células T CD8 ${ }^{+}$ leva à maior susceptibilidade ao desafio micobacteriano (KAUFMANN, 2001).

O efeito micobactericida do IFN- $\gamma$ produzido pelos linfócitos $\mathrm{T}$ envolve a produção de óxido nítrico e outros radicais reativos de nitrogênio e oxigênio na célula hospedeira (macrófagos), mas alguns achados recentes demonstram que isso pode explicar apenas parcialmente a ação desta citocina (MACMICKING et al., 2003). Foi demonstrado que os efeitos do IFN- $\gamma$ ocorrem pelo menos em parte através de uma GTPase, a LRG-47 (GUTIERREZ et al., 2004). A LRG-47 é um membro da família de GTPases de resistência que foi descrita como sendo um dos principais fatores de proteção relacionado ao $M$. tuberculosis. Foi ainda demonstrado que a LRG-47 pode participar na indução de autofagia dependente de IFNy e que esse processo está relacionado à diminuição da viabilidade da micobactéria intracelular (GUTIERREZ et al., 2004).

O IFN- $\gamma$ é crítico em mediar a proteção, como confirmado por observações no modelo murino. A relevância 
de tais respostas protetoras do IFN- $\gamma$ é reforçada por informações de que mutações naturais em genes humanos que codificam IFN $\gamma$-R, IL-12, IL-12R ou STAT-1 e conseqüentemente levam à imunidade mediada por IFN- $\gamma$, aumentam a susceptibilidade a infecções por micobactérias (SAHIRATMADJA et al., 2007; HWANG et al., 2007; OTTENHOFF et al., 2003).

Ao mesmo tempo em que uma resposta de IFN- $\gamma$ parece estar associada com o controle da doença, diversos estudos demonstraram que a tuberculose humana está associada com o aumento da resposta Th2 e o envolvimento das citocinas IL-4 e IL-13, bem como a secreção de IgE dependente de IL-4 (YONG et al., 1989; SEAH et al., 2000). Além disso, a infecção pulmonar leva à produção aumentada das citocinas IL-10 e TGF- $\beta$ que promovem um ambiente no qual as células do sistema imunológico recém-recrutadas ficam refratárias aos sinais de ativação imunológica (BONECINI-ALMEIDA et al., 2004).

Outra importante citocina no controle da tuberculose é o TNF-alfa. Foi observado que pacientes tratados com antagonistas de TNF-alfa exibem um aumentado risco de doença micobacteriana, o que coloca o TNF-alfa como uma importante molécula na defesa do hospedeiro contra patógenos intracelulares (BOURIKAS et al., 2008; JOLOBI, 2007; STRADY et al., 2006).

Nos últimos anos muitos estudos têm procurado caracterizar moléculas de $M$. tuberculosis para inclusão em uma nova vacina contra a tuberculose e novos métodos diagnóstico. Nossos estudos preliminares demonstraram que a produção de IFN- $\gamma$ em pacientes com tuberculose estava aumentada em resposta aos antígenos 85B e ferritina quando comparados aos controles (ANTAS et al., 2002; CARDOSO et al., 2002). Estudos anteriores demonstraram que o ESAT-6 (Early secreted antigenic target 6-kDa) é um dos antígenos micobacterianos determinantes para a produção de linfócitos $\mathrm{T}$ produtores de IFN- $\gamma$. O antígeno ESAT-6 foi descrito como tendo um grande número de epítopos de células B e T que são reconhecidos pelos soros de pacientes com tuberculose, implicando o seu papel na indução de memória imune contra o bacilo (HARBOE et al., 1998; MUSTAFÁ et al., 1998). Os genes do ESAT-6 e CFP-10 (culture filtrate protein $10 \mathrm{kDa}$ ) estão codificados na região RDl do genoma de micobactérias. Esta região está presente apenas no $M$. tuberculosis, $M$. africanum e $M$. bovis (complexo $M$. tuberculosis) e em algumas micobactérias do ambiente ( $M$. kansasii, M. marinum e $M$. szulgai) estando ausente na vacina BCG e na maioria das micobactérias ambientais, o que torna essas moléculas importantes alvos de estudo para avaliar a capacidade imunomodulatória e ainda sua capacidade para discriminar indivíduos com a forma latente da doença.

$\mathrm{Na}$ tentativa de avaliar a resposta imune antígeno - específica em uma população no município do Rio de Janeiro, foi avaliada a produção de IFN- $\gamma$ em sobrenadantes de culturas de células mononucleares de sangue periférico (PBMCs) de pacientes com tuberculose nãoresistente e de pacientes com TBMR. Foi observado que os pacientes com TBMR apresentam baixa produção de IFN- $\gamma$ em resposta ao ESAT-6 se comparada aos pacientes com tuberculose não-resistente antes e após o tratamento (FORTES et al., 2005). Esses dados observados em pacientes TBMR no Rio de Janeiro estão de acordo com outros estudos da literatura, que demonstram que PBMCs de pacientes MDR-TB apresentam diminuída resposta de TNF-alfa a antígenos de $30 \mathrm{kDa}$ de $M$. tuberculosis. Neste estudo, os autores demonstraram que a resposta diminuída aos antígenos de $M$. tuberculosis por pacientes portadores de TBMR, é modulada pela citocina IL-10 (LEE et al., 2003). Recentemente, um estudo avaliando a resposta imune de pacientes TBMR a antígenos lipídicos revelou que as PBMCs e células T $\mathrm{CD}^{+}{ }^{+}$destes pacientes responderam menos aos antígenos lipídicos do que as células de indivíduos PPD-positivos. Em adição, foi observado que a produção de IL-4 em resposta aos antígenos lipídicos estava aumentada nos pacientes TBMR, com baixa resposta de IFN- $\gamma$, quando comparados aos indivíduos PPD-positivos, sugerindo que as células $\mathrm{T} \mathrm{CD}^{+}$de pacientes TBMR polarizam para uma resposta Th2 (SHAHEMABADI et al., 2007).

Apesar de diversos estudos apontarem para a dicotomia Th1 (IFN- $\gamma$ ) -Th2 (IL-4, IL-10) como sendo parâmetros associados à resistência-susceptibilidade à doença, uma maior compreensão sobre os mecanismos imunorregulatórios envolvidos no controle da tuberculose deve ser buscada. Ainda que inúmeras evidências apontem para a importância do IFN- $\gamma$ no controle da doença em modelos experimentais e em pacientes com tuberculose não-resistente, PARK et al. (2007) demonstraram que em pacientes com TBMR avançada ou crônica, o tratamento subcutâneo com IFN- $\gamma$ não resultou em melhora dos parâmetros clínicos, radiológicos, microbiológicos ou imunológicos, sugerindo que outros fatores podem estar envolvidos no controle da doença nesses pacientes. Desse modo, a compreensão sobre os mecanismos imunológicos envolvidos nas diferentes formas clínicas e temporais da doença pode auxiliar no desenvolvimento de medidas profiláticas e mesmo terapêuticas mais efetivas contra a doença.

\section{Novas estratégias de controle da tuberculose}

O teste utilizado no diagnóstico da infecção latente é o Tuberculin Skin Test (TST), ou teste intradérmico com o derivado protéico purificado. Pode-se afirmar que o TST tem pobre especificidade e não é fácil de ser avaliado (BLANC et al., 2007; MENZIES, 1999). Dois testes diagnósticos baseados na produção de IFN- $\gamma$ - o T-SPOT.TB e o Quantiferon-TB Gold - foram licenciados recentemente. Esses testes requerem somente uma amostra de sangue e seus resultados não são dependentes do examinador. Sua especificidade é maior do que a do TST porque eles não apresentam reação cruzada com a vacinação para BCG e com a maioria das micobactérias ambientais, uma vez que esses testes utilizam os antígenos ESAT-6 e CFP-10. Também no diagnóstico da tuberculose latente, esses ensaios apresentam melhor correlação com a exposição ao $M$. tuberculosis do que o TST. No entanto, a capacidade de detectar TB nesses dois testes é reduzida em pacientes com HIV, especial- 
mente aqueles em tratamento (BLANC et al., 2007). Além disso, não se sabe se esses ensaios serão facilmente realizados em países com pouca estrutura. Desse modo, novos métodos de diagnóstico são ainda necessários.

Entre as novas propostas para diagnóstico destacamse o MGIT (Mycobacterium Growth Indicator Tube) que permite o rápido crescimento e a detecção da bactéria com uma redução no tempo de detecção. Outra proposta é a Capilia TB, um teste que confirma a presença de bacilo nas culturas em 15 minutos. Também estão sendo desenvolvidos testes com o objetivo de avaliar resistência a drogas em pacientes com esfregaço positivo, como é o caso do FASTPlaque-Response Test e o Genotype MTBDR Test, desenvolvidos pelo Geneva-based Foundation for Innovative New Diagnostics (FIND) em parceria com instituições privadas como o Biotec Laboratories e Hain Lifescience $G m b H$, respectivamente. O conhecimento da genética molecular tem levado a outras alternativas que asseguram um diagnóstico mais rápido; no entanto, deve-se levar em consideração que esses testes moleculares dependem de alto investimento na compra de equipamentos, bem como no treinamento da equipe, o que pode levar a dificuldades de implantação de tais métodos em países em desenvolvimento (YEW et al., 2008).

Apesar da existência de medicamentos eficazes no controle da doença, o elevado tempo de tratamento leva muitas vezes à falta de adesão a terapia, o que culmina na ocorrência de casos de resistência aos fármacos. Assim, tem-se buscado medicamentos que reduzam o período de tratamento, uma vez que a redução do tempo de tratamento incorrerá na maior adesão dos pacientes, transmissão reduzida de TB e menor resistência aos fármacos, com conseqüente diminuição no número de óbitos decorrentes da doença.

Dois novos medicamentos encontram-se em fase de testes clínicos. O primeiro é o moxifloxacin, um fármaco já no mercado, desenvolvido pela Bayer, em fase III de testes clínicos para tuberculose. Além deste, outros fármacos se encontram em estudo, o nitroimidazol (PA824), desenvolvido em parceria entre a TB Alliance e a Chiron Corporation nos Estados Unidos, está em fase I de testes clínicos, representando um painel promissor, que há muito tempo não se via, desde a descoberta da rifampicina há mais de 40 anos (GARWOOD, 2007).

A vacina BCG foi introduzida em 1921 e é ainda hoje a única vacina contra a tuberculose. A BCG é capaz de prevenir as formas de tuberculose em crianças; no entanto, a sua eficácia em adultos é altamente variável (NABESHIMA et al., 2005) e estudos demonstram que a re-vacinação em jovens e adultos não aumenta o nível de proteção (RODRIGUES et al., 2005). Durante os últimos anos, diversos candidatos a vacinas foram identificados e, ao menos oito vacinas entraram em testes clínicos, que estão ainda em fase inicial (GUPTA et al., 2007). A caracterização do M. tuberculosis H37Rv por métodos de proteômica revelou várias proteínas secretadas que poderiam ser investigadas. Entretanto, o desenvolvimento de novas vacinas deve levar em consideração os efeitos de marcadores centrais para obter um melhor painel da regulação da resposta imune (WIKER et al., 2006), desse modo, uma maior compreensão dos mecanismos imunológicos relacionados à tuberculose e ao desenvolvimento da TBMR podem auxiliar no desenvolvimento de medidas profiláticas mais efetivas. É preciso que o acúmulo de informações obtidas nas pesquisas básicas contribuam efetivamente para o desenvolvimento de estratégias efetivas no controle da doença. Além disso, TEIXEIRA et al. (2007) ressaltam o fato de que ainda que uma vacina mais eficaz do que o BCG seja levada ao mercado, esta não será capaz de prevenir a progressão da doença entre os mais de 2 milhões de indivíduos já infectados, o que reforça a importância de prosseguirem as pesquisas por ferramentas diagnósticas e terapêuticas adequadas ao controle da doença.

\section{Referências bibliográficas}

ANTAS, P.R. et al. Kinetics of T cell-activation molecules in response to Mycobacterium tuberculosis antigens. Memórias do Instituto Oswaldo Cruz, v.97, p.10971099, 2002.

BALIZA, M. et al. High frequency of resistance to drugs isoniazid and rifampicin among tuberculosis cases in the city of Cabo de Santo Agostinho, an urban area in Northeastern Brazil. Revista da Sociedade Brasileira de Medicina Tropical, v.41, p.11-16, 2008.

BLANC, P. et al. New blood tests for diagnosis of infection with Mycobacterium tuberculosis. Revue des Maladies Respiratoires, v.24, p.441-452, 2007.

BONECINI-ALMEIDA, M.G. et al. Down-modulation of lung immune responses by interleukin-10 and transforming growth factor beta (TGF- $\beta$ ) and analysis of TGFbeta receptors I and II in active tuberculosis. Infection and Immunity, v.72, p.2628-2634, 2004.

BOURIKAS, L.A. et al. Disseminated tuberculosis in a Crohn's disease patient on anti-TNF alpha therapy despite chemoprophylaxis. Gut. v.57, p 425, 2008.

CARDOSO, F.L. et al. T-cell responses to the Mycobacterium tuberculosis-specific antigen ESAT-6 in Brazilian tuberculosis patients. Infection and Immunity, v.70, p.6707-6714, 2002.

CDC. Centers for Disease Control and Prevention. Extensively drug-resistant tuberculosis - United States, 1993-2006. MMWR Morbidity and Mortality Weekly Report, v.56, p.250-253, 2007.

COOPER, A.M. et al. Interleukin 12 (IL-12) is crucial to the development of protective immunity in mice intravenously infected with Mycobacterium tuberculosis. Journal of Experimental Medicine, v.186, p.39-45, 1997.

DALCOLMO, M.P. et al. Estudo de efetividade de esquemas alternativos para o tratamento da tuberculose multirresistente no Brasil. Journal of Pnemology, v.25, p.70-77, 1999.

DALCOLMO, M.P.; ANDRADE, M.K.; PICON, P.D. Multiresistant tuberculosis in Brazil: history and control. Revista de Saúde Pública, v.41, p.34-42, 2007. 
DEHEINZELIN, D. et al. Fatores preditivos de abandono de tratamento por pacientes com tuberculose. Revista do Hospital das Clinicas, São Paulo, v.51, p.131-135, 1996.

DINIZ, L. et al. Efetividade do tratamento da tuberculose em oito capitais brasileiras. Boletin de Pneumologia Sanitária, v.3, p.6-18, 1995.

ESPINAL, M.A. et al. Global trends in resistance to antituberculosis drugs. World Health Organization - Internacional Union against Tuberculosis and Lung Disease Working Group on Anti-tuberculosis Drug Resistance Surveillance. New England Journal of Medicine, v.344, p.1294-1303, 2001.

FORTES, A. et al. Detection of in vitro interferongamma and serum tumour necrosis factor-alpha in multidrug-resistant tuberculosis patients._Clinical and Experimental Immunology, v. 141, p.541-548, 2005.

GARWOOD, P. New tools for an old disease. Bulletin of the World Health Organization, v.85, p.331-332, 2007.

GRANICH, R.M. et al. Multi-drug resistance among persons with tuberculosis in California, 1994-2003. Journal of the American Medical Association, v.293, p.2732-2739, 2005

GUPTA, U. D.; KATOCH, V. M.; MCMURRAY, D.N. Current status of TB vaccines. Vaccine. v.25, p.37423751, 2007.

GUTIERREZ, M.G. et al. Autophagy is a defense mechanism inhibiting BCG and Mycobacterium tuberculosis survival in infected macrophages. Cell, v.119, p.753766, 2004.

HARBOE, M. et al. B-cell epitopes and quantification of the ESAT-6 protein of Mycobacterium tuberculosis. Infection and Immunity, v.66, p.717-723, 1998.

HERRMANN, J.L. et al. The role of human dendritic cells in tuberculosis: protector or non-protector? Revue des Maladies Respiratoires, v.23, p.6S21-6S28, 2006.

HIJJAR, M.A.; CAMPOS, H.S.; FEITOSA, J.V.P. Tuberculose. In: COURA, J.R. (Ed.\}. Dinâmica das doenças infecciosas e parasitárias. Rio de Janeiro: Guanabara Koogan, 2005.

HWANG, J.H. et al. Polymorphisms of interferon-gamma and interferon-gamma receptor 1 genes and non-tuberculous mycobacterial lung diseases.Tuberculosis, v.87, p.166-171, 2007.

ISEMAN, M.D. Treatment of multidrug-resistant tuberculosis. New England Journal of Medicine, v.329, p.784-791, 1993.

JOLOBE, O.M. Anti-TNFalpha treatment and reactivation of latent tuberculosis. Lancet, v.370, p.27-28, 2007.
KAUFMANN, S.H. How can immunology contribute to the control of tuberculosis? Nature Reviews Immunology, v.1, p.20-30, 2001.

KRITSKI, A.L. et al.. Taxa de abandono do tratamento antituberculose. Pulmão, v.1 1, p.9-15, 2002.

LEE, J.S. et al. The production of tumour necrosis factoralpha is decreased in peripheral blood mononuclear cells from multidrug-resistant tuberculosis patients following stimulation with the 30-kDa antigen of Mycobacterium tuberculosis. Clinical \& Experimental Immunology, v.132, p.443-449, 2003.

LOCHT, C. et al. How a different look at latency can help to develop novel diagnostics and vaccines against tuberculosis. Expert Opinion on Biological Therapy, v.7, p.1-13, 2007.

MACMICKING, J.D.; TAYLOR, G.A.; MCKINNEY, J.D. Immune control of tuberculosis by IFN-gamma-inducible LRG-47. Science, v. 302, p.654-659, 2003.

MENZIES, D. Interpretation of repeated tuberculin tests: boosting, conversion and reversion. American Journal of Respiratory and Critical Care Medicine, v.159, p 15-21, 1999.

MITCHISON, D.A. Drug resistance in tuberculosis. European Respiratory Journal, v.25, p.376-379, 2005.

MUSTAFA, A.S. et al. Comparison of antigen-specific T-cell responses of tuberculosis patients using complex or single antigens of Mycobacterium tuberculosis. Scandinavian Journal of Immunology, v.48, p.535-543, 1998.

NABESHIMA, S. et al. Serum antibody response to tuberculosis-associated glycolipid antigen after BCG vaccination in adults. Journal Infection Chemotherapy, v.1 1, p.256-258, 2005.

OTTENHOFF, T.H. et al. Human deficiencies in type1 cytokine receptors reveal the essential role of type- 1 cytokines in immunity to intracellular bacteria. Advances in Experimental Medicine and Biology, v.531, p.279-94, 2003.

PARK, S.K. et al. Subcutaneously administered interferon-gamma for the treatment of multidrug-resistant pulmonary tuberculosis. Internal Journal Infection Disease, v.11, p.434-440, 2007.

RAVIGLIONE, M.C.; PIO, A. Evolution of WHO policies for tuberculosis control, 1948-2001. Lancet, v.350, p.624-629, 2002.

RODRIGUES, L.C. et al. Effect of BCG revaccination on incidence of tuberculosis in school-aged children in Brazil; the BCG-REVAC cluster-randomised trial. Lancet, v.366, p. 1290-1295. 2005.

RUSSELL, D.G. Phagosomes, fatty acids and tuberculosis. Nature Cell Biology, v.5, p.776-778, 2003.

RUSSELL, D.G. Who puts the tubercle in tuberculosis? Nature Reviews Microbiology, v.5, p.39-47, 2007. 
SAHIRATMADJA, E. et al. Association of polymorphisms in IL-12/IFN-gamma pathway genes with susceptibility to pulmonary tuberculosis in Indonesia. Tuberculosis, v.87, p.303-31 1, 2007.

SEAH, G.T.; SCOTT, G.M.; ROOK, G.A. Type 2 cytokine gene activation and its relationship to extent of disease in patients with tuberculosis. Journal of Infectious Diseases, v.181, p.385-389, 2000.

SHAHEMABADI, A.S. et al. Evaluation of T cell immune responses in multidrug-resistant tuberculosis (MDR-TB) patients to Mycobacterium tuberculosis total lipid antigens._Clinical Experimental Immunology, v.149, p.285-294, 2007.

SNIDER, DE J.R. et al. Infection and disease among contacts of tuberculosis cases with drug-resistant and drug-susceptible bacilli. American Review of Respiratory Disease, v.132, p.125-132, 1985.

STEINGART, K.R. et al.Sputum processing methods to improve the sensitivity of smear microscopy for tuberculosis: a systematic review. Lancet Infectious Diseases, v.6, p.664-674, 2006.

STRADY, C. et al. Tuberculosis during treatment by TNFalpha-inhibitors. Presse Medicale, v.35, p.17651772, 2006

TEIXEIRA, H.C.; ABRAMO, C.; MUNK, M. E. Diagnóstico imunológico da tuberculose: problemas e estratégias para o sucesso. Jornal Brasileiro de Pneumologia, v.33, p.323-334, 2007.

TELLES, M.A. et al. A population-based study of drug resistance and transmission of tuberculosis in an urban community. International Journal of Tuberculosis and Lung Disease, v.9, p.970-976, 2005.

URBANCZIK, R. Present position of microscopy and of culture in diagnostic mycobacteriology. Zentralblatt fur Bakteriologie and Mikrobiologie and Hygiene, v.260, p.81-87, 1985

VALWAY, S. E. et al. Multidrug-resistant tuberculosis in the New York State prison system, 1990-1991.Journal on Infectious Diseases, v.170, p.151-156, 1994.
VANACORE, P. et al. GISTA-SIMIT study group. Drugresistant tuberculosis in HIV-infected persons: Italy 1999-2000. Infection, v.32, p.328-332, 2004.

VERGNE, I. et al. Cell biology of Mycobacterium tuberculosis phagosome. Annual Review of Cell and Developmental Biology, v.20, p.367-394, 2004.

WIKER, H.G. et al. Vaccine approaches to prevent tuberculosis. Scandinavian Journal Immunology, v.64, p.243-250, 2006.

WORLD HEALTH ORGANIZATION. Global tuberculosis control: surveillance, planning, financing. Geneva: WHO, 2007.

WORLD HEALTH ORGANIZATION. The global plan to stop TB: 2005-2006. Geneva: WHO, 2006.

WORLD HEALTH ORGANIZATION. Global tuberculosis control: surveillance, planning, financing: WHO Report 2005. Geneva: WHO, 2005.

WORLD HEALTH ORGANIZATION. Stop TB. tuberculosis and children. Geneva, Switzerland: WHO, 2004.

WORLD HEALTH ORGANIZATION. TB deaths reach historic levels. WHO Press, 1996. p.1-3.

YEW, W.W.; CHAU, C.H. Drug-resistant tuberculosis in the 1990s. European Respiratory Journal, v. 8, p.1184-1192, 1995.

YEW, W.W.; LEUNG, C.C. Management of multidrugresistant tuberculosis: update 2007. Respirology, v. 13, p.21-46, 2008.

YONG, A.J. et al. Total and anti-mycobacterial IgE levels in serum from patients with tuberculosis and leprosy. Tubercle, v.70, p.273-279, 1989.

ZAGER, E.M.; MCNERNEY, R. Multidrug-resistant tuberculosis. BMC Infectious Diseases, v.8, p. 1-5, 2008.

ZIGNOL, M. et al. Global incidence of multidrug-resistant tuberculosis. Journal of Infection Disease, v. 194, p. 479-485, 2006. 


\section{Sobre os autores}

\section{Roberta Olmo Pinheiro}

Possui graduação em Farmácia pela Universidade Federal do Rio de Janeiro (1998). Mestrado (2000) e Doutorado em Ciências (2004) realizados no Laboratório de Imunofarmacologia, do Instituto de Biofísica Carlos Chagas Filho (IBCCF/UFRJ). Pós-Doutorado no Laboratório de Biologia Imunitária (IBCCF/UFRJ). Atualmente é Pesquisadora Visitante do Laboratório de Hanseníase, do Pavilhão de Hanseníase (IOC/Fiocruz). Tem experiência nas áreas de Parasitologia e Imunologia, com ênfase em Imunologia, atuando principalmente nos seguintes temas: doenças infecciosas, imunorregulação, morte celular e vacinas.

\section{Margareth Pretti Dalcolmo}

Graduada em Medicina pela Escola de Medicina da Santa Casa de Misericórdia de Vitória. Doutorado em Medicina (Pneumologia) pela Universidade Federal de São Paulo e Residência-médica pelo Hospital Raphael de Paula Souza. Tem experiência na área de Saúde Coletiva, com ênfase em Pneumologia. Atuando principalmente nos seguinte tema: Tuberculose. 